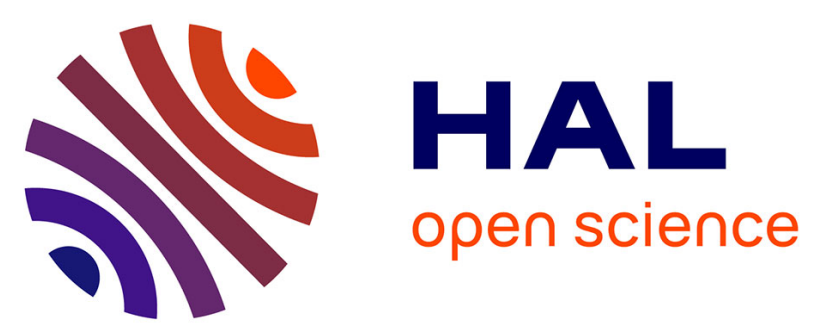

\title{
De La Bastide de Beauvezet à la Verrerie entre la fin du Moyen Âge et le XVIIe siècle: artisanat du verre dans la forêt de Cadarache (Bouches-du-Rhône)
}

Gerald Bonnamour, Christophe Vaschalde, Mathilde Tissot

\section{- To cite this version:}

Gerald Bonnamour, Christophe Vaschalde, Mathilde Tissot. De La Bastide de Beauvezet à la Verrerie entre la fin du Moyen Âge et le XVIIe siècle: artisanat du verre dans la forêt de Cadarache (Bouchesdu-Rhône). Archéologie du Midi Médiéval, 2011, 29 (1), pp.227-240. 10.3406/amime.2011.2018 . hal-02131873

\section{HAL Id: hal-02131873 \\ https://hal.science/hal-02131873}

Submitted on 16 May 2019

HAL is a multi-disciplinary open access archive for the deposit and dissemination of scientific research documents, whether they are published or not. The documents may come from teaching and research institutions in France or abroad, or from public or private research centers.
L'archive ouverte pluridisciplinaire HAL, est destinée au dépôt et à la diffusion de documents scientifiques de niveau recherche, publiés ou non, émanant des établissements d'enseignement et de recherche français ou étrangers, des laboratoires publics ou privés. 


\title{
De La Bastide de Beauvezet à la Verrerie entre la fin du Moyen
} Âge et le XVIle siècle : artisanat du verre dans la forêt de Cadarache (Bouches-du-Rhône)

\author{
Gérald Bonnamour, Christophe Vaschalde, Mathilde Tissot
}

\section{Citer ce document / Cite this document :}

Bonnamour Gérald, Vaschalde Christophe, Tissot Mathilde. De La Bastide de Beauvezet à la Verrerie entre la fin du Moyen Âge et le XVIle siècle : artisanat du verre dans la forêt de Cadarache (Bouches-du-Rhône). In: Archéologie du Midi médiéval. Tome 29, 2011. pp. 227-240;

doi : https://doi.org/10.3406/amime.2011.2018

https://www.persee.fr/doc/amime_0758-7708_2011_num_29_1_2018

Fichier pdf généré le 22/03/2019 


\title{
Resumen
}

De La Gran Finca de Beauvezet a la Cristalería entre finales de la Edad Media y el siglo XVII : artesanado del vidrio en el bosque de Cadarache (Bouches-du-Rhône).

El proyecto de construcción del reactor nuclear Iter, a proximidad del centro de investigación del Comisariado para la Energía Atómica (CEA) de Cadarache, está al origen de una prescripción de excavaciones arqueológicas preventivas en el lugar llamado la Verrerie (Saint-Paul-Lès-Durance, Bouches-du-Rhône). Llevada a cabo en diciembre de 2007 sobre una superficie de $400 \mathrm{~m}^{2}$, la intervención arqueológica realizada por la sociedad Arkemine, bajo la dirección de Gérald Bonnamour, abarcó los vestigios construidos de la «bastide" o gran finca de Beauvezet y los vestigios enterrados de una cristalería. En complemento de la excavación arqueológica, unas investigaciones en los registros de archivo han permitido apreciar mejor la historia de la finca y del bosque de Cadarache pero también las actividades relacionadas con los recursos silvestres entre la Edad Media y el periodo moderno. Una cristalería era en actividad en la finca de Beauvezet a finales del siglo XVII, durante unos diez años. Esta actividad de cristalería está documentada por escritos pero también por la arqueología preventiva.

\begin{abstract}
From the Bastide de Beauvezet to the Verrerie between the end of the Middle Ages and the XVIIth century, glass-works crafts in the Cadarache forest (Bouches-du-Rhône).

The project of construction of the Iter nuclear reactor, nearby the research centre of the French Atomic Energy Authority (CEA) in Cadarache, is the origin of a preventive archaeological excavations prescription at the place called the Verrerie (Saint-Paul-Lès-Durance, Bouches-duRhône). Carried on in December of 2007 over an area of $400 \mathrm{~m}^{2}$, the archaeological intervention implemented by the Arkemine company, under the direction of Gérald Bonnamour, has brought to the light the built vestiges of the manor of Beauvezet and the buried vestiges of a glasshouse. In addition to the archaeological excavations, some investigations in the records have allowed to understand better the history of the estate and of the Cadarache forest but also the activities related to the woodsy resources between the Middle Ages and the modern era. A glasshouse has been active at the fortified town of Beauvezet at the end of the XVIlth century, for some ten years. This glassworks activity is documented by written sources but also by the preventive archaeology.
\end{abstract}

\section{Résumé}

Le projet de construction du réacteur nucléaire Iter, à proximité du centre de recherche du Commissariat à l'Énergie Atomique (CEA) à Cadarache, est à l'origine d'une prescription de fouille d'archéologie préventive au lieu-dit la Verrerie (Saint-Paul-Lès-Durance, Bouches-du-Rhône). Effectuée en décembre 2007 sur une surface de $400 \mathrm{~m}^{2}$, l'intervention archéologique réalisée par la société Arkemine, sous la direction de Gérald Bonnamour, a portée sur les vestiges bâtis de la bastide de Beauvezet et sur les vestiges enfouis d'une verrerie. En complément de la fouille archéologique, des recherches en archives ont permis de mieux apprécier l'histoire du domaine et de la forêt de Cadarache mais également des activités en lien avec les ressources sylvestres entre le Moyen Age et la période moderne. Une verrerie était en activité à la bastide de Beauvezet à la fin du XVIle siècle, durant une dizaine d'années. Cette activité verrière est documentée par les sources écrites, mais également par l'archéologie préventive.

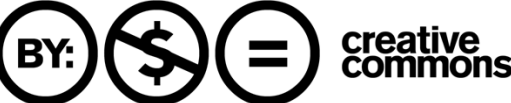




\title{
De La Bastide de Beauvezet à la Verrerie entre la fin du Moyen Âge et le XVII siècle : artisanat du verre dans la forêt de Cadarache (Bouches-du-Rhône)
}

\author{
Gérald BONNAMOUR*, Christophe VASCHALDE* ${ }^{* *}$ Mathilde TISSOT**
}

Le projet de construction du réacteur nucléaire Iter, à proximité du centre de recherche du Commissariat à l'Énergie Atomique (CEA) à Cadarache, est à l'origine d'une prescription de fouille d'archéologie préventive au lieu-dit la Verrerie (Saint-Paul-Lès-Durance, Bouches-du-Rhône). Effectuée en décembre 2007 sur une surface de $400 \mathrm{~m}^{2}$, l'intervention archéologique réalisée par la société Arkemine, sous la direction de Gérald Bonnamour, a portée sur les vestiges bâtis de la bastide de Beauvezet et sur les vestiges enfouis d'une verrerie. En complément de la fouille archéologique, des recherches en archives ont permis de mieux apprécier l'histoire du domaine et de la forêt de Cadarache mais également des activités en lien avec les ressources sylvestres entre le Moyen Age et la période moderne. Une verrerie était en activité à la bastide de Beauvezet à la fin du XVII siècle, durant une dizaine d'années. Cette activité verrière est documentée par les sources écrites, mais également par l'archéologie préventive.

Mots-clés : bastide, verrerie, four de recuit, ressources sylvestres, forêt

\section{INTRODUCTION}

Les vestiges de la bastide de Beauvezet se trouvent au lieu dit la Verrerie, au sein de la forêt de Cadarache à Saint-Paul-Lès-Durance (Fig. 1). La construction du réacteur nucléaire Iter a nécessité la réalisation d'un diagnostic préventif dont l'emprise couvrait plus de 100 ha de la forêt domaniale, puis d'une fouille de la bastide et des vestiges d'un atelier verrier qui s'y était installé durant le seconde moitié du XVII siècle. Malgré une courte durée de vie de l'atelier, ce changement de fonction a été si marquant que le toponyme a été modifié, la bastide de Beauvezet étant devenue « la Verrerie » (1) (Fig. 2).

Le castrum de Cadarache est mentionné pour la première fois en 1089 (Guérard 1857, charte $\mathrm{n}^{\circ} 436$ ), lorsqu'un certain Guillaume Geoffroy donne aux moines bénédictins de l'abbaye Saint-Victor de Marseille un manse et la sixième partie du castrum. L'église SaintMichel, située à proximité du château, relevait de l'abbaye Saint-André de Villeneuve au XII ${ }^{c}$ siècle (Albanès 1891, 58). En 1343, elle possède encore le statut d'église paroissiale du village qui s'est formé autour du château, mais ce dernier est déclaré dépeuplé en 1378 suite aux épidémies et aux guerres (Mouton 2008, 83). À partir du

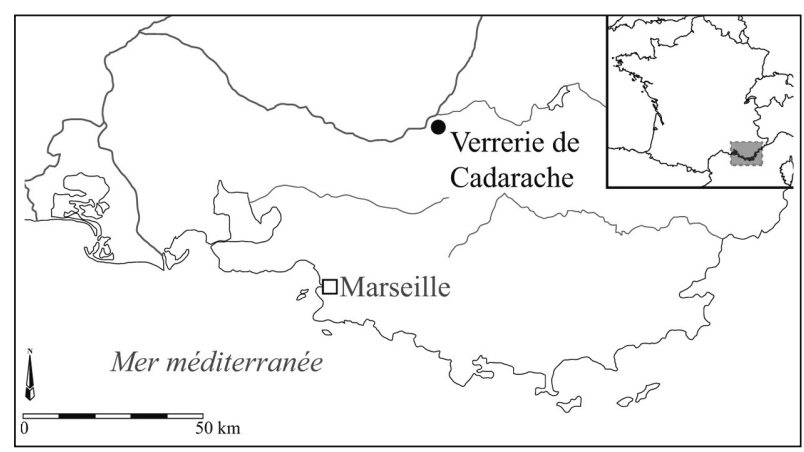

Fig. 1 : Carte de situation.

$\mathrm{XVI}^{e}$ siècle, le domaine appartient successivement aux familles de Savoie-Tende, de Saluces et de Valbelle.

Les résultats de l'étude du site ont été obtenus grâce à la mobilisation des méthodes de plusieurs disciplines, principalement l'archéologie et l'histoire. Les fouilles ont été complétées par un relevé du bâti des vestiges de la bastide et de l'atelier verrier, tandis que le travail en archives a porté sur une cinquantaine de registres des notaires de Saint-Paul-Lès-Durance et Meyrargues entre

\footnotetext{
* Responsable d'opération, Arkemine SARL, La Talarde, La Plaine de Clairac, 26760 Beaumont-lès-Valence.

** Dépouillement des registres des notaires de Saint-Paul-lès-Durance et Meyrargues (13), Aix-Marseille Université, LA3M - UMR 7298.

*** Arkemine SARL, La Talarde, La Plaine de Clairac, 26760 Beaumont-lès-Valence.

'En 1778, lorsqu'est effectuée la levée de la carte de Cassini dans le secteur, le toponyme « Beauvezet » est encore signalé. Lors de la vente du domaine comme bien national le 16 germinal an V (5 avril 1797), il est question de la « Bastide de Beauvezet, dite la Veyrière » (Moulin 1910, vol. 3, 622). Enfin, le toponyme est définitivement remplacé par la dénomination « La Verrerie » sur le cadastre napoléonien (1813), sur la carte d'État-major et, plus tard, sur la carte I.G.N. au 25/000
} 


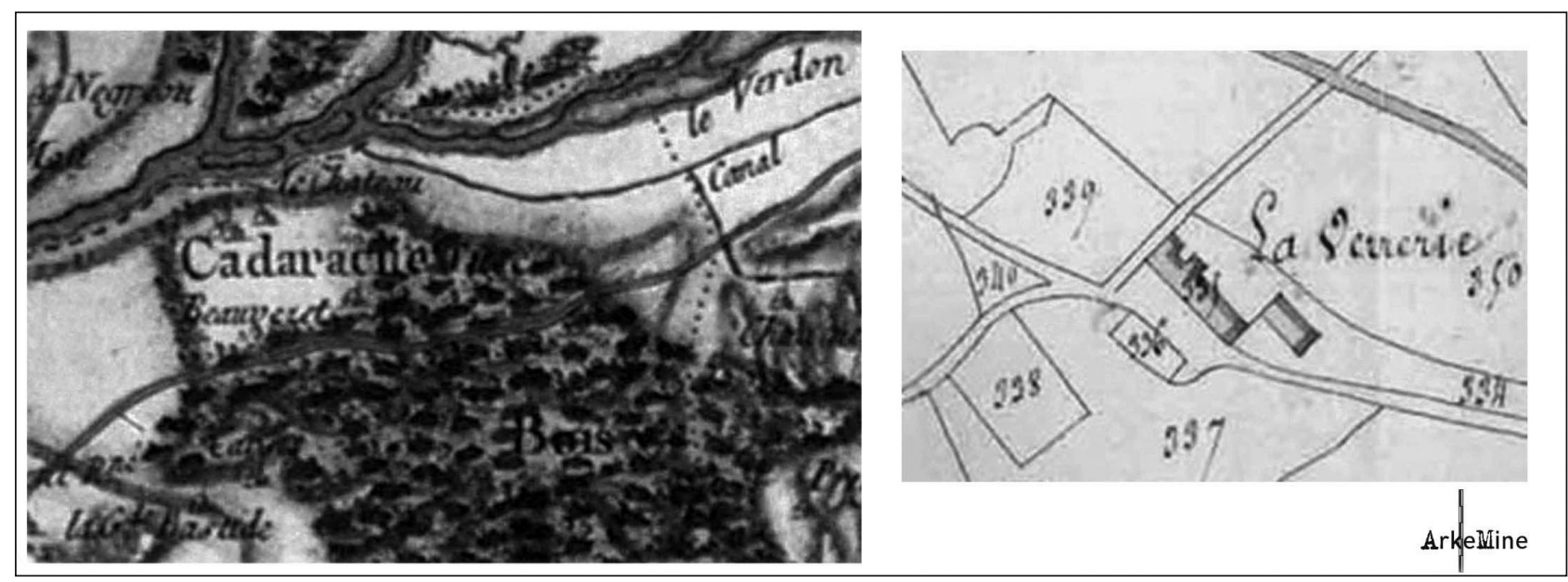

Fig. 2 : Carte de Cassini à gauche et cadastre napoléonien à droite.

1580 et 1692 (2), auxquels s'ajoutent des documents issus de la Chambre des Eaux et Forêts de la Cour des comptes, aides et finances du Parlement de Provence (3), ou de la Cour de justice des seigneurs de Cadarache (4). L'histoire du site est ainsi connue au travers de plus d'une centaine de documents écrits, dont de nombreux contrats de mègerie et de mise en fermage.

Avant l'implantation du travail du verre, la forêt de Cadarache était déjà un lieu d'activités artisanales. L'exploitation du bois alimente entre autres le charbonnage, connu grâce aux autorisations accordées aux maréchaux-ferrants du château de Cadarache pour alimenter leur forge, mais aussi la tuilerie de Saint-Paul-LèsDurance lorsque le seigneur de Cadarache y fait cuire une fournée de tuiles (5). Plusieurs fours à chaux sont installés dès le $\mathrm{XVII}^{\mathrm{e}}$ siècle. Un four a d'ailleurs été retrouvé au moment du diagnostic archéologique (Martin, 2007 ; Martin, Fournier 2007).

Si le toponyme la Verrerie ne laisse pas de doute sur la vocation artisanale du site, des vestiges bâtis en élévation ou enfouis identifiés par la fouille renvoient à l'existence de la bastide de Beauvezet. Les sources écrites et archéologiques documentent une activité verrière qui a contribué à modifier la bastide physiquement mais également dans son statut.

\section{La bastide de Beauvezet avant les verriers : entre agriculture et pastoralisme}

\section{Le bâti existant}

En plus du château et de ses terres, le terroir de Cadarache est composé de plusieurs fermes, dont les bastides de Carcès et de Beauvezet. De cette dernière, seul subsiste en élévation le mur nord M201 (Fig. 3 et 4). Le parement externe de ce mur de façade est devenu, suite à la construction de la halle des verriers vers le nord, un parement interne de l'édifice construit en pierres, encore en élévation au moment de la fouille. À l'intérieur du bâtiment, ce mur est en partie masqué au sud-est par une pièce voutée sur laquelle se trouve une autre pièce formant un étage accessible par un escalier situé contre la façade extérieure à l'est du bâtiment. Au moment de la fouille, une construction en béton et parpaings masquait le parement sud du mur.

Les parties visibles du mur M201 permettent d'apprécier le soin apporté à la construction de la bastide. Le parement nord est constitué pour sa fraction supérieure d'un petit appareil de moellons grossièrement équarris et approximativement assisés, liés avec un mortier de chaux rouge orangé (Fig. 3). Dans sa partie inférieure, le type d'appareil ne change pas, mais le mortier à granulométrie fine devient gris et inclut des nodules de chaux. Cet ensemble correspond à une reprise en sous-œuvre postérieure à la construction du mur.

Outre les ouvertures récentes et bien caractérisées, trois fenêtres contemporaines de la construction du mur M201 ont été mises en évidence. Ces ouvertures étaient condamnées au moment de l'intervention. La première est une fenêtre à meneau (Fig. 4) dont les caractéristiques et la présence de moulures décoratives démontrent que le parement nord du mur est en fait le parement externe. L'appui, les piédroits, le meneau et la traverse sont constitués de blocs taillés de calcaire jaune. Cette fenêtre a été arasée au moment de la mise en place de la toiture

\footnotetext{
${ }^{2}$ AD Bouches-du-Rhône, séries 399 E et 414 E. Avant 1580, aucun registre de notaire ayant officié à Saint-Paul n'est conservé, et la gestion du domaine de Cadarache et de ses bastides reste inconnue.

${ }_{3}^{3}$ AD Bouches-du-Rhône, série B.

${ }^{4}$ AD Bouches-du-Rhône, 6B 4141 (1712-1713).

${ }^{5}$ Charbonnage et tuileries ne sont absolument pas associés dans l'exploitation de la forêt (Vaschalde en cours). Sauf dans quelques rares exemples médiévaux découverts en Provence, le charbonnage utilise surtout des grosses buches pour produire de la matière charbonneuse qui a pour propriété principale de concentrer la chaleur en un seul point dans la forge, ce qui est fondamental pour le travail du métal. Au contraire, les fours de tuiliers en tout cas dans le Midi méditerranéen- utilisent des fagots de broussailles ou, éventuellement, des petites buches, non utilisés par les charbonniers ou les bucherons, pour faire une flamme qui fait monter la chaleur dans le four, et donc dans la charge. L'usage du charbon dans les fours de tuiliers n'existe pas avant la Révolution industrielle.
} 


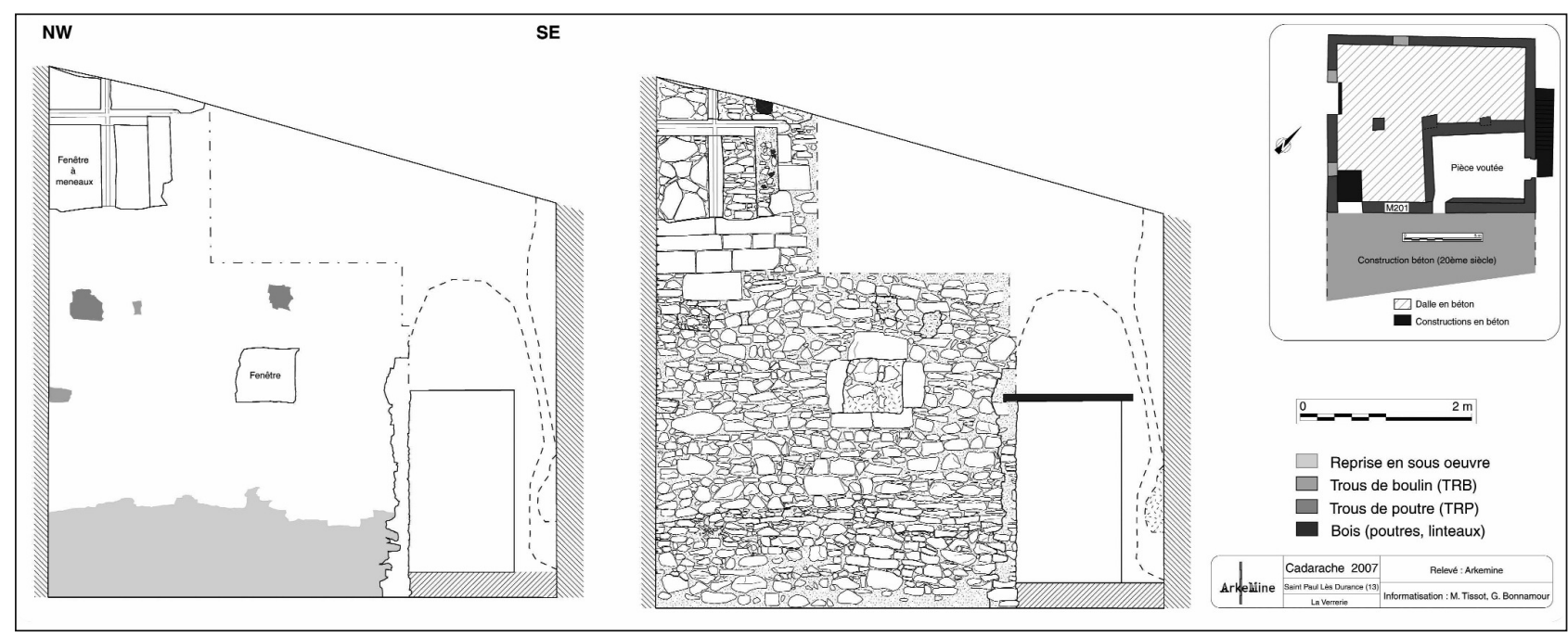

Fig. 3 : Relevé d'élévation du mur sud du bâtiment construit en pierre à l'intérieur du bâtiment (M201).

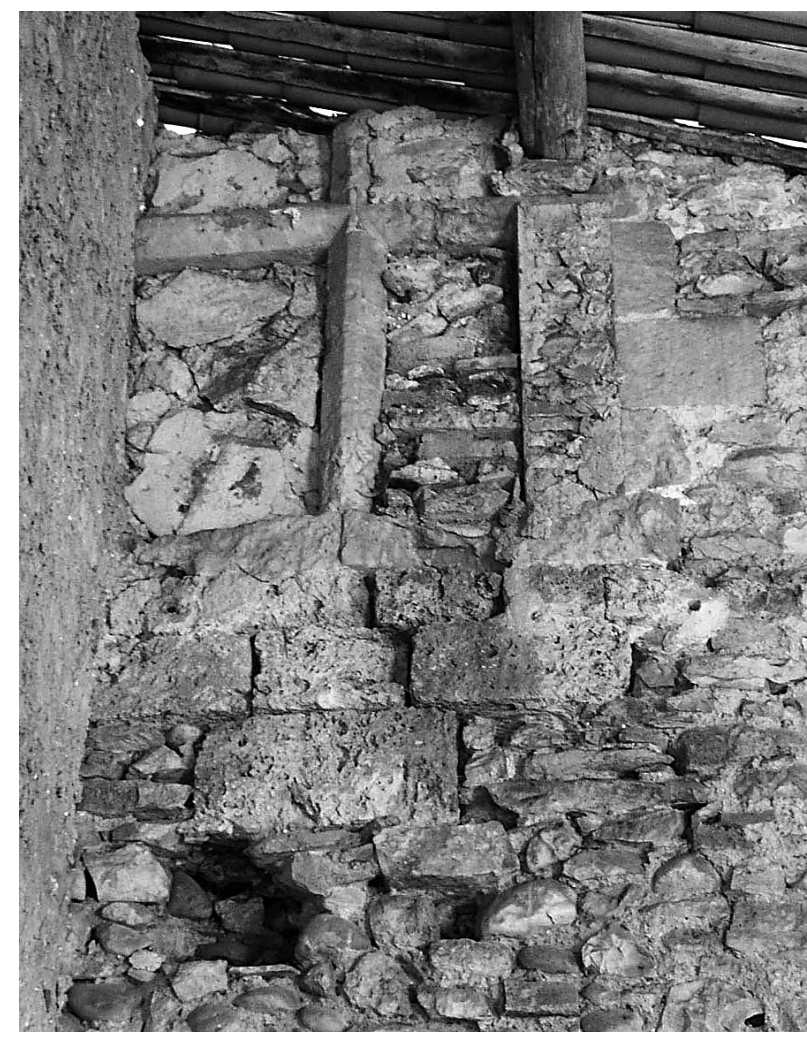

Fig. 4 : Fenêtre à meneau en place dans le mur sud du bâtiment construit en pierre à l'intérieur du bâtiment. Cliché G. Bonnamour (Arkemine).

actuelle, faisant ainsi disparaître le linteau. Cette lacune indique que la hauteur du mur M201 était initialement plus importante, et que la bastide possédait un étage. La deuxième fenêtre est une petite ouverture carrée de $62,5 \mathrm{~cm}$ de côté. Les pierres de taille constituant son encadrement sont constituées d'un travertin grisâtre très vacuolaire. La troisième ouverture est visible au-dessus d'une porte, au sud de la pièce voûtée. D'après les observations, cette porte se trouve d'ailleurs à l'emplacement d'une porte plus ancienne, peut-être contemporaine de la construction de la bastide.

Lors de la fouille, une arase de mur a été découverte à l'ouest du bâtiment, dans l'alignement du mur M201. Les deux maçonneries étant similaires, ceci laisse supposer qu'il s'agissait d'un seul et même mur qui s'étendait vers l'ouest.

\section{Une bastide issue de la troisième génération de défrichements médiévaux?}

Grâce aux archives, la gestion de la bastide de Beauvezet est connue surtout entre les années 1580 et 1630 environ. Plusieurs types d'actes notariés ont été recensés dans les registres. Mais à partir de 1630 environ, le fonds des notaires de Saint-Paul ne livre plus de documents, si ce n'est au moment de l'installation des verriers. Un changement d'habitude dans la pratique du notariat par les seigneurs de Cadarache explique peutêtre ce constat.

Aux XVI ${ }^{\mathrm{e}}$ et $\mathrm{XVII}{ }^{\mathrm{e}}$ siècles, l'ensemble du territoire de Cadarache est mis en valeur par un système de mise en fermage, très répandu dans la Provence d'époque moderne (Amouric 1993). Le seigneur passe régulièrement des contrats d'arrentement pour l'ensemble du terroir. Le rentier (6), à qui échoit donc l'exploitation et l'entretien des terres et propriétés pour plusieurs années, passe à son tour très fréquemment des contrats de mégerie (7) pour chacune des bastides de Beauvezet ou de Carcès, contrats passés plus spécialement dans le cadre des activités pastorales. Toutefois, ce dernier type

\footnotetext{
${ }^{6}$ Plusieurs rentiers de Cadarache ont été recensés : Claude Constant en 1581 puis de 1597 à 1601 ; Michel Beaumont en 1585, devenu co-rentier en 1587 avec $\mathrm{M}^{\circ}$ Jean Perrin ; Billes Bassier et Esprit Berneaud en 1608; Louis Verran en 1609; Clément Reynaud en 1613; André Cornillon entre 1620 et 1624. ${ }^{7}$ Bail à cheptel (Coulet 1988). Plusieurs mégiers de Beauvezet ont été recensés : Marc Vincent, originaire de Vinon-sur-Verdon en 1585 ; Jean et Thomas Gautier, originaires d'Oppède, en 1608 ; Antoine Brunel, originaire de Ginasservis en 1620 ; Honnorat Hugon, originaire de Saint-Julien-leMontagnet, en 1624.
} 
d'acte est parfois établi directement entre le seigneur et le mégier. Dans tous les cas, les contrats comportent des clauses obligeant le mégier à rendre les biens en bon état, à assurer de bonnes récoltes et, plus généralement, à gérer l'exploitation «en bon père de familhe » (8).

En 1608, le domaine de Beauvezet est dit « bastide et pasturière » (9), ce qui témoigne parfaitement de la double vocation agricole et pastorale de cette exploitation implantée à proximité immédiate des collines boisées de la forêt de Cadarache.

\section{La céréaliculture}

L'agriculture pratiquée est essentiellement céréalière. Les semences sont fournies par le seigneur, et le mégier doit les rendre sur l'aire du château chaque année après les récoltes (10). Les techniques de culture ne sont jamais explicitées. Une quittance (11) passée en 1608 donne les quantités de grains que l'ancien rentier de Cadarache devait rendre au seigneur et au nouveau rentier (Fig. 5). Ce document donne donc un aperçu des récoltes et des proportions tenues par chaque espèce de céréale, même s'il faut garder à l'esprit que ne sont indiquées que les quantités rendues, qui ne représentent donc qu'une part minime des récoltes totales, et dont l'ampleur est inconnue. Les deux céréales les plus semées sont le seigle $(41 \%)$ et le froment $(37,3 \%)$. La part de l'avoine s'élève à 18,8\% du lot versé, mais sa présence marque, sans surprise pour l'époque moderne, l'utilisation de la rotation des cultures, déjà en place en Provence depuis au moins le XIV ${ }^{\mathrm{e}}$ siècle (Coulet 1988, Drendel 1991). L'orge n'est présente qu'en toute petite quantité $(2,9 \%)$, et servait notamment à nourrir les cochons (12).

En plus des céréales, des légumes sont plantés, comme les fèves. En 1608, le seigneur en récupère

\begin{tabular}{|l|r|r|r|r|r|}
\hline \multicolumn{1}{|c|}{ Céréales } & Charges & \multicolumn{1}{c|}{ Panaux } & Civadiers & Décalitrcs & \multicolumn{1}{c|}{$\%$} \\
\hline Avoine & 32 & 3 & 2 & 1035,2 & $18,8 \%$ \\
\hline Froment & 64 & 3 & & 2057,6 & $37,3 \%$ \\
\hline Orge & 4 & 9 & 3 & 159,2 & $2,9 \%$ \\
\hline Seigle & 70 & 6 & 2 & 2260,8 & $41,0 \%$ \\
\hline Total & $\mathbf{1 7 0}$ & $\mathbf{2 1}$ & 7 & $\mathbf{5 5 1 2 , 8}$ & $\mathbf{1 0 0 , 0} \%$ \\
\hline
\end{tabular}

Fig. 5 : Quantités de céréales versées par l'ex-rentier de Cadarache en 1608.
1 civadier et demi, soit environ 1,2 décalitres. Un jardin est signalé à côté de la bastide (13).

La rente seigneuriale n'est jamais constituée d'une part de récolte de raisin. Pourtant, les mégiers sont tenus d'entretenir des vignes (14), mais tous les fruits leur appartiennent (15), et rien n'est jamais dit sur le vin qui est probablement produit. Tout au plus, ils doivent fournir des cabusses au seigneur, c'est-à-dire des plans de jeunes vignes créés par marcottage dans le but de renouveler les cépages (16). En 1585, le mégier Marc Vincent ne jouit que des deux tiers de la vigne de « La Beste » (toponyme non situé), qu'il doit partager avec le mégier de la bastide de Carcès (17). En 1624, la vigne confiée à Honnorat Hugon est située non pas près de la bastide, mais à côté du château (18). Ce partage et cette dispersion des parcelles semblent montrer que la part de la viticulture dans l'activité de la bastide est très certainement réduite, constat assez courant dans les exploitations de ce type en Provence depuis la fin du Moyen Age (Coulet 1988, 192 ; Amouric 1993, 328). Il n'est pas inutile non plus de remarquer l'absence totale de mentions d'arboriculture fruitière.

\section{Le pastoralisme}

Dans ses activités agricoles, la bastide de Beauvezet est presque exclusivement tournée vers la céréaliculture. Pourtant, elle constitue aussi le centre d'une forte activité de pastoralisme. Rares sont les prés mentionnés (19), mais la dépaissance est basée sur la mise à profit des « herbages » de la forêt, probablement des formations de landes, ainsi que de la glandée. C'est ainsi qu'est exploitée la majorité de la surface du terroir de Cadarache, couvert aux deux tiers de bois comme le signalait en 1730 une enquête de la Chambre des Eaux et Forêts (20).

Entre 1581 et 1624 , plusieurs documents fournissent des indications sur les effectifs de troupeaux qui circulent dans la forêt de Cadarache, et dont certains sont conduits par les mégiers de Beauvezet (Fig. 6). Dans la bastide même, les troupeaux de chèvres et moutons sont les plus nombreux, et peuvent atteindre 540 têtes. Ils servent notamment à la production de laine et de fromage (21), dont une partie doit être versée chaque année au seigneur. Les troupeaux de porcs installés à Beauvezet sont quant à eux de taille plus réduite, et n'ex-

\footnotetext{
${ }^{8}$ AD Bouches-du-Rhône, 399 E 253, $\mathrm{f}^{\circ} 198$ (12 septembre 1585$) ; 273$, feuillet $1620, \mathrm{f}^{\circ} 19 \mathrm{v}^{\circ}$ et 24 (16 mars 1620$) ; 274, \mathrm{f}^{\circ} 29 \mathrm{v}{ }^{\circ}(12$ avril 1624$)$; $274, f^{\circ} 49$ (24 avril 1624).

${ }^{9}$ AD Bouches-du-Rhône, 399 E 267, fo 58 (8 avril 1608).

${ }^{10}$ AD Bouches-du-Rhône, 399 E 273, feuillet 1620, fo 19 v (16 mars 1620) ; 274, fo 49 (24 avril 1624).

${ }^{11}$ AD Bouches-du-Rhône, 399 E 267, fo 58 (8 avril 1608).

${ }^{12}$ AD Bouches-du-Rhône, 399 E 273, feuillet 1620, fo 19 v (16 mars 1620).

${ }^{13}$ AD Bouches-du-Rhône, 399 E 253, fo 198 (12 septembre 1585).

${ }^{14}$ AD Bouches-du-Rhône, 399 E 253, fo 198 (12 septembre 1585) ; 273, feuillet 1620 , fo $19 \mathrm{v}^{\circ}$ (16 mars 1620$)$; $274, \mathrm{f}^{\circ} 49$ (24 avril 1624$)$.

${ }^{15}$ AD Bouches-du-Rhône, 399 E 273, feuillet $1620, \mathrm{f}^{\circ} 19 \mathrm{v}^{\circ}(16$ mars 1620).

${ }^{16}$ AD Bouches-du-Rhône, 399 E 253, fo 198 (12 septembre 1585).

${ }^{17}$ AD Bouches-du-Rhône, 399 E 253, f 198 (12 septembre 1585).

${ }^{18}$ AD Bouches-du-Rhône, 399 E 274, f ${ }^{\circ} 49$ (24 avril 1624).

${ }^{19}$ AD Bouches-du-Rhône, 399 E 253, fo 198 (12 septembre 1585).

${ }^{20}$ AD Bouches-du-Rhône, B 6185, p. 88.

${ }^{21}$ AD Bouches-du-Rhône, 399 E 253, f 198 (12 septembre 1585).
} 


\begin{tabular}{|c|c|c|c|c|c|c|c|c|c|c|}
\hline & 1581 & $1585^{*}$ & 1587 & 1597 & 1605 & 1606 & 1609 & $1619^{*}$ & $1620^{*}$ & $1624^{*}$ \\
\hline Cochons & 152 & 60 & & 57 & & 6 & & & $60-90$ & 6 \\
\hline $\begin{array}{c}\text { Moutons } \\
\text { et chèvres }\end{array}$ & & 60 & 135 & & 750 & 90 & 870 & 540 & 52 & 120 \\
\hline * : annécs dont les effcctifs concernent la bastidc de Bcauvczet) &
\end{tabular}

Fig. 6: Effectifs de troupeaux signalés dans la forêt de Cadarache entre 1581 et 1624 dans les registres des notaires de Saint-Paul.

cèdent jamais 60 têtes. Certaines années, le nombre de porcs est parfois très réduit, et pourrait refléter un élevage à but strictement domestique. Chèvres, moutons et porcs ne sont pas les seuls animaux élevés. Les contrats de mègeries signalent régulièrement des poules, pour lesquelles le seigneur prélève des chapons (22). Les bovins (23) et mulets (24) servent pour le labour des terres céréalières notamment, pour lequel la ferme est équipée de charrues (25), mais produisent aussi du fumier.

L'ensemble des activités agricoles et pastorales se traduisent matériellement par l'aménagement d'espaces aux fonctions spécialisées dans ou autour du bâtiment de la bastide. Ainsi, les différents contrats évoquent tour à tour une étable pour les bœufs, un jas pour les troupeaux, des greniers ou une aire de battage.

\section{Une fondation à la fin du Moyen Age?}

Le recours aux méthodes de la fouille archéologique, du relevé du bâti et du dépouillement des sources historiques permet de dresser un portrait de la bastide de Beauvezet entre le début des années 1580 et l'installation des verriers en 1666. Ce portrait, assez complet par certains aspects, souffre de deux lacunes principales : le manque de vestiges appartenant aux bâtiments antérieurs à l'arrivée des verriers, et l'absence de toute documentation permettant une analyse de la géographie ancienne du terroir (livres terriers ou inventaires). Mais, de par son implantation géographique et ses activités de production agricole et de mise en valeur de l'espace inculte par le pastoralisme, cette bastide s'inscrit dans la droite ligne de la génération des bastides agricoles et pastorales qui se sont multipliées en Provence à partir de la fin du $\mathrm{XIV}^{\mathrm{e}}$ siècle. Une comparaison peut être effectuée sans trop de risques avec les établissements décrits par N. Coulet dans la campagne aixoise des derniers siècles du Moyen Age (Coulet 1988, 192). Comme à Beauvezet, les bastides, édifices quadrangulaires construits avec un étage, y étaient installées en marge des terroirs cultivés, et, dans une entreprise de défrichement et/ou de remise en culture des confins, alliaient céréaliculture et pastoralisme principalement, les autres formes de productions agricoles (vigne, arboriculture) étant délaissées. Mais cette comparaison se limite-t-elle à la seule fonctionnalité de l'établissement? Avec toute la prudence qui doit être adoptée dans un cas comme celui de Beauvezet, des hypothèses peuvent être avancées pour tenter de cerner l'époque de la construction de la bastide. Tout d'abord, la fenêtre à meneau peut constituer un terminus post quem. Le décor chanfreiné de la croisée n'apparaît en Provence qu'au milieu du $\mathrm{XV}^{\mathrm{e}}$ siècle, tandis que, dans le Lubéron et autour d'Aix, la croisée elle-même ne se répand vraiment en milieu rural qu'à partir des années 1480 (Bernardi 1996). De par son architecture, son implantation et sa fonction, la bastide de Beauvezet pourrait très bien avoir été édifiée dans un $\mathrm{XV}^{\mathrm{e}}$ siècle avancé, bien que rien n'interdise d'envisager une édification dans le courant du XVI ${ }^{\mathrm{e}}$ siècle. Reste à expliquer les motivations d'une telle entreprise. Comme dans le reste de la Provence, la recherche de nouvelles terres cultivables y est certainement pour beaucoup. Mais le contexte local est à prendre en compte également. Le village de Cadarache est déserté depuis au moins 1378, et toute forme de communauté villageoise a définitivement disparu. Après cela, le seigneur apparaît comme le seul gestionnaire de la mise en valeur de la terre et sera, certainement, à l'initiative de la construction de la bastide de Beauvezet, mais aussi de celle de Carcès. Une nouvelle forme d'exploitation du terroir se met donc en place, uniquement fondée sur des cellules d'habitat et d'exploitation dispersées.

\section{Quand la bastide de Beauvezet devient Verrerie}

\section{L'installation des verriers au travers des actes notariés}

En octobre 1667, le seigneur de Cadarache Léon de Valbelle passe un contrat d'arrentement (26) avec les frères Antoine et Melchion Desferres, deux verriers nobles (Fig. 7). À cette date, une année s'est déjà écoulée depuis la construction de la verrerie à Beauvezet, qui avait d'abord été exploitée par Joseph Desferres, oncle des deux nouveaux rentiers. Mais pendant cette première année, l'atelier ne semble pas avoir fonctionné puisque le contrat de 1667 stipule bien qu'il revient à Antoine et Melchion de construire un four. Outre quelques clauses ayant trait au fonctionnement même de la verrerie, le contrat prévoit de donner la jouissance des biens de la bastide de Beauvezet aux artisans (habitation, jardin, puits, terres labourables...), pour eux comme pour leur bétail. Ils sont d'ailleurs autorisés à faire paître six pourceaux dans le bois (27), mais peuvent aussi chasser les dimanches et jours de fête, ou encore aller pêcher dans la Durance. Pendant les premiers mois, Antoine et Melchion travaillent avec leur oncle Joseph, ainsi qu'avec François Desferres, fils de ce dernier.

\footnotetext{
${ }^{22}$ AD Bouches-du-Rhône, 399 E 253, fo 198 (12 septembre 1585) ; 273, feuillet 1620 , fo $19 \mathrm{v}^{\circ}(16$ mars 1620$) ; 274, \mathrm{f}^{\circ} 49$ (24 avril 1624$)$.

${ }^{23}$ AD Bouches-du-Rhône, 399 E 273, feuillet $1620, f^{\circ} 19 \mathrm{v}^{\circ}$ (16 mars 1620); 274, fo 49 (24 avril 1624).

${ }^{24}$ AD Bouches-du-Rhône, 399 E 274, fo 49 (24 avril 1624).

${ }^{25}$ AD Bouches-du-Rhône, 399 E 273, feuillet 1620, fo $19 \mathrm{v}^{\circ}$ (16 mars 1620); 274, fo 49 (24 avril 1624).

${ }^{26}$ AD Bouches-du-Rhône, 414 E 89, fo $703 \mathrm{v}^{\circ}$ (30 octobre 1667).

${ }^{27}$ La pratique du pastoralisme par les verriers est rappelée dans un autre document quelques mois plus tard (AD Bouches-du-Rhône, 399 E 319 , f 260

$\mathrm{v}^{\circ}(22$ décembre 1668).
} 


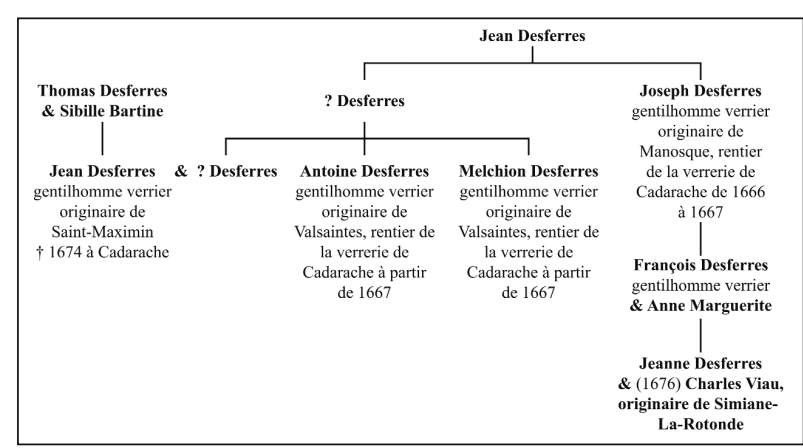

Fig. 7 : Généalogie des Desferres, verriers de la bastide de Beauvezet (C. Vaschalde).

L'installation des Desferres dans ce secteur de la Provence n'est pas due au hasard. La production du verre est une activité fortement consommatrice en combustible végétal. L'arrentement de 1667 précise d'ailleurs certaines modalités de gestion des bois, mais reste muet sur l'exploitation des ressources géologiques. Dans le Sud-est de la France, les textes provençaux de la fin du Moyen Age font clairement apparaître que la préoccupation principale des verriers est l'approvisionnement en combustible (Foy 1988, 45). Ainsi, le manque de bois est également la principale cause de départ ou de déplacement des ateliers, et donc des verriers. Le choix d'implantation tient compte à la fois de la contrainte du combustible, et, dans une moindre mesure, de la présence des ressources minérales nécessaires à la fabrication du verre (Amouric, Foy 1992). Le terroir anciennement déserté de Cadarache est un secteur idéal de ce point de vue, puisqu'il offre à la fois l'argile pour les creusets, la chaux qui entre dans la composition des verres, tandis que la silice se trouve dans le lit de la Durance, en grande partie constitué d'alluvions intégrant des sables, des graviers et des galets de quartz ainsi que des roches riches en silice. Cependant, un terroir riche en ressources et peu exploité n'est pas unique en Provence. L'installation de trois verreries successives à Roquefeuille à Pourrières (83) entre la fin du $\mathrm{XV}^{\mathrm{c}}$ et le XVIII ${ }^{\circ}$ siècle paraît suivre la même logique (Foy, Vallauri 1989). Un secteur forestier comme celui de Cadarache apparaît donc comme idéal pour les verriers. Mais si la gestion du domaine et de l'atelier est entièrement confiée aux artisans, le seigneur se réserve des moyens d'action non négligeables sur l'approvisionnement en combustible. Les coupes sont réglées en quartiers, dont le premier est décrit trop sommairement dans le contrat pour pouvoir toucher au cœur de cette gestion ; mais, surtout, il choisit lui-même un « homme de travail » chargé de procéder à la récolte et à la livraison du combustible. Les clauses vont jusqu'à interdire la vente des cendres du four au tisonnier.
Ce dernier, tenté de se constituer une source de revenus secondaires en les vendant aux paysans comme engrais, pourrait peut-être brûler plus de combustible que nécessaire, ce que le seigneur cherche à éviter.

Les premiers rentiers de la verrerie sont tous des Desferres (Fig. 7), patronyme rappelant fortement la famille Ferry ou de Ferre qui a donné nombres de verriers provençaux depuis la fin du Moyen Age, et à laquelle ceux de Cadarache sont certainement apparentés (Foy 1988). Ils sont originaires de Valsaintes, de Manosque et de Saint-Maximin, où se trouvent déjà des centres de production de verre. Au nombre de quatre en 1667, les verriers de Cadarache ne sont déjà plus que trois l'année suivante lorsque Joseph se retire définitivement (28). En septembre 1670, deux ans avant le terme de leur contrat, Melchion et Antoine Desferres sousarrentent la verrerie en faveur de noble François Descrivas, originaire du Bac, pour la durée restante du contrat passé avec le seigneur Léon de Valbelle. François Descrivas est alors assisté d'un tisonnier nommé Antoine Roux. À partir de cette date, les frères Antoine et Melchion Desferres n'exercent plus leur art à Cadarache, mais perçoivent la rente convenue par contrat (29), et Melchion y réside encore en 1674 (30).

Le dépouillement des registres n'a pas permis d'établir la durée de la présence de François Descrivas à la tête de la verrerie de Cadarache. En avril 1671, il reconnait devoir un salaire de 60 livres à Jean Desferres qui travaille pour lui (31), mais après cette date il n'apparaît plus dans les registres. Les trois dernières années d'activité sont marquées par la mort de Jean Desferres en 1674 (32), et un conflit avec un tisonnier nommé Alexandre Barruel qui, ayant quitté la verrerie subitement, provoque un arrêt momentané de la production (33). Après 1676, toute trace est perdue de l'atelier dans les registres des notaires de Saint-Paul.

\section{L'organisation spatiale de la verrerie}

L'installation des verriers se traduit sur le terrain par l'aménagement d'une halle au nord de la bastide, s'appuyant contre le mur M201 (Fig. 8). Cet espace, qui mesure environ $9 \mathrm{~m}$ de largeur pour $10,50 \mathrm{~m}$ de longueur, couvre une surface d'environ $95 \mathrm{~m}^{2}$. Le relevé du bâti, qui n'a pu être fait que sur une partie des élévations à cause de la forte contrainte liée à la sécurité du chantier, a mis en évidence des maçonneries constituées d'un petit appareil de moellons et de galets grossièrement équarris, très approximativement assisés, liés avec un mortier de chaux beige clair incluant des nodules de chaux centimétriques. Ces murs sont chaînés aux angles par des moellons de moyen appareil, équarris et à face dressée, la

\footnotetext{
${ }^{28}$ AD Bouches-du-Rhône, 399 E 319, fo 209 (3 juin 1668).

${ }^{29}$ AD Bouches-du-Rhône, 399 E 320, fo 205 (26 janvier 1675). Melchion Desferres apparaît également comme débiteur dans deux autres reconnaissances de dettes auprès d'habitants du village de Saint-Paul : 399 E 319, f 614 (25 avril 1671) ; 320, fo $204 \mathrm{v}^{\circ}$ (26 janvier 1675$)$.

${ }^{30}$ AD Bouches-du-Rhône, 399 E 320, fo 23 (9 février 1674).

${ }^{31}$ AD Bouches-du-Rhône, 399 E 319, fo 612 (17 avril 1671).

${ }^{32}$ AD Bouches-du-Rhône, registres paroissiaux de Saint-Paul-Lès-Durance (24 juillet 1674).

${ }^{33}$ AD Bouches-du-Rhône, 399 E 320, f $45 \mathrm{v}^{\circ}$ (24 juillet 1674).
} 


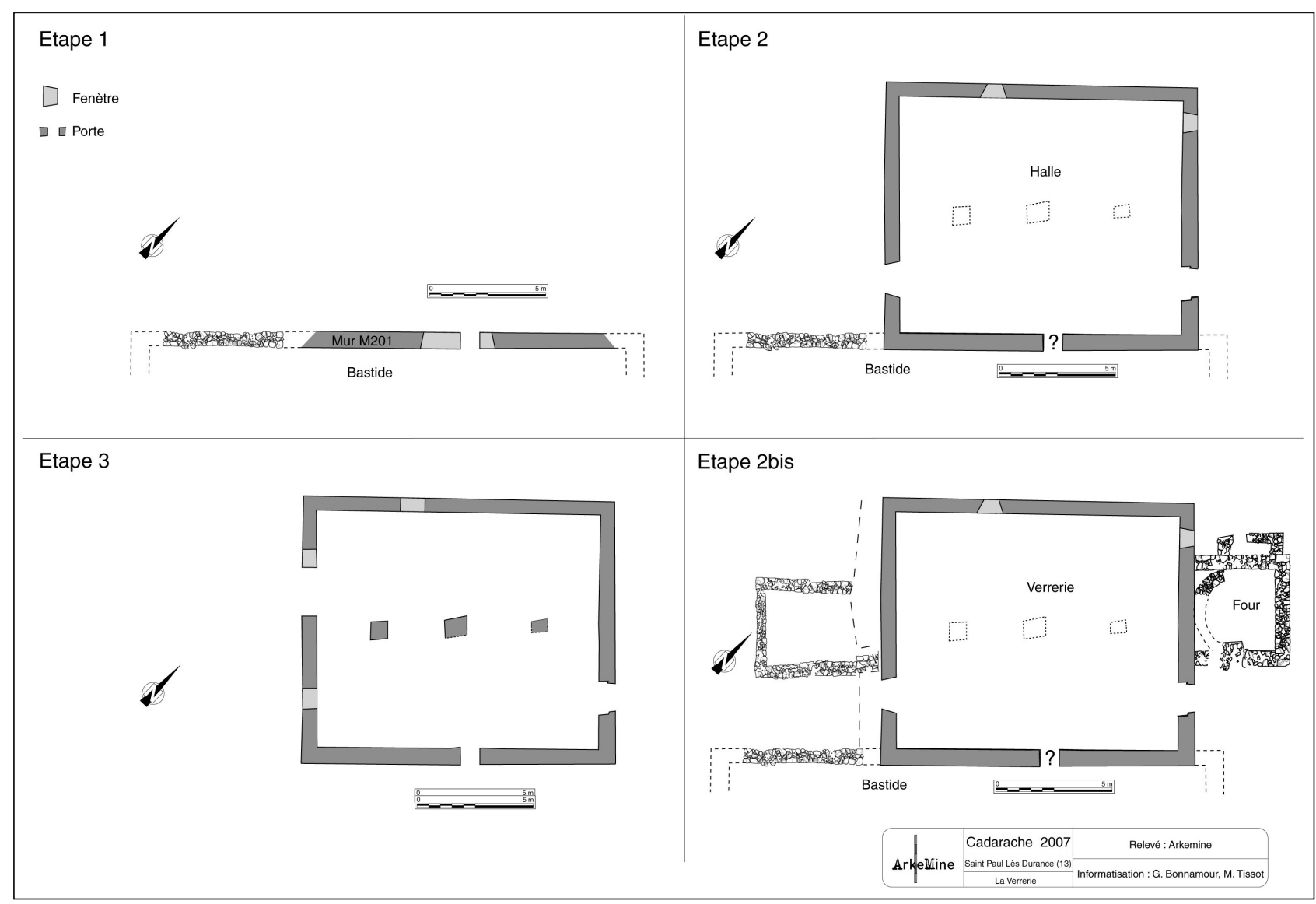

Fig. 8 : Les phases de constructions du bâtiment construit en pierre et s'appuyant contre et au nord de la bastide de Beauvezet.

plupart taillés dans un " tuf » calcaire. La halle était accessible par deux portes se faisant face, situées l'une à l'est, et l'autre à l'ouest, cette dernière ayant été murée par la suite. Les encadrements sont construits en moyen appareil de pierres de taille, avec linteaux en bois. Chaque mur de la halle était également percé d'une fenêtre, dont les appuis ne sont pas tous à la même altitude. Toutes trois étaient murées au moment de la fouille, et seulement deux d'entre elles, au nord et à l'est, étaient munies d'un ébrasement. À l'intérieur, deux niveaux de trous de boulins ont été mis en évidence $(302,15$ et 304 $\mathrm{m}$ NGF). Le mur septentrional de la halle conservait également des trous de poutres, renforcés à leur base par un appareil de moellons calcaires équarris, similaires à d'autres repérés sur le parement nord du mur M201. Ces trous témoignent de l'aménagement d'un étage. À l'intérieur, le seul sondage, réalisé dans la pièce voûtée, n'a malheureusement dévoilé aucun vestige d'occupation archéologique.

À cet ensemble s'ajoutent deux extensions, très arasées, l'une à l'ouest ( 6 x 4,50 m) et l'autre à l'est $(4,75 \times 4 \mathrm{~m})$. Les maçonneries sont identiques à celles des murs de la halle, ce qui témoigne d'une construction contemporaine. L'extension orientale, bordée d'un petit espace maçonné quadrangulaire $(2,75 \times 0,95 \mathrm{~m})$, abritait les vestiges d'un four d'environ 2,60 $\mathrm{m}$ de diamètre, identifié comme un four de recuit (cf. infra). À l'ouest, autour de la cellule formée par l'autre extension, des amas de chaux ont été identifiés (Fig. 8). À l'intérieur, des niveaux d'effondrement recouvraient un niveau d'abandon qui piégeait des couches cendreuses à la base de la stratigraphie. Dans ce secteur, de nombreux fragments de creusets ont été découverts.

Malgré l'absence de vestiges dans la halle, les restes mis au jour à l'ouest et à l'est semblent révéler une organisation spatiale qui suit les différentes étapes de la chaine opératoire technique. Autour de l'extension occidentale, les matières premières auraient été stockées en vue de leur préparation. À l'opposé de la halle se trouveraient ensuite les dernières étapes du travail des verriers, autour du four de recuit.

La fouille ayant été circonscrite au nord du mur de façade de la bastide M201, le périmètre de cette dernière n'a fait l'objet d'aucune intervention. L'habitat des verriers n'est donc pas connu. De la vie quotidienne, seul subsiste le matériel céramique à usage domestique. Les formes renvoient aux pratiques culinaires ou alimentaires : marmite à anse cannelée de Vallauris, assiette à marli à verni blanc, plat vernissé rouge, plusieurs oreilles d'écuelles vernissées rouge ou jaune tachées de vert, cruche à pâte rouge. Les décors montrent que les productions identifiées proviennent des officines provençales, notamment de la vallée de l'Huveaune (notamment un fond de plat décoré d'une tulipe brune sur engobe banc tâché de vert) ou de Vallauris. Une partie du matériel est 
attribuable au XVII ${ }^{\mathrm{e}}$ siècle (Abel, Amouric 1995 ; Amouric et al. 1999, 98-100).

Après le départ des verriers, le bâtiment a subi de nouvelles modifications pour être transformé en entrepôt et en bergerie. Au moment de ces aménagements, l'étage existant a été supprimé.

\section{Le four}

Les archives consultées ne font mention que d'un seul four, construit probablement vers la fin de l'année 1667, et à nouveau mentionné en 1671. Pourtant, la succession des étapes de la chaîne opératoire technique du verre est telle que plusieurs fours sont généralement construits. Dans les contrats d'arrentement, les notaires, et surtout les contractants, ne se soucient guère du nombre de four, qui est une affaire qui ne concerne que les seuls artisans verriers (Commandré en cours). Les archives démontrent que des tisonniers travaillaient pour les verriers de Cadarache ( $c f$. infra). Ils étaient probablement chargés de l'entretien du four. Quant aux verriers, ils s'occupaient certainement de la préparation des matières premières et du mélange introduit dans le creuset, mais également de la mise en forme et du façonnage des objets.

Les vestiges du four ont été retrouvés dans une des extensions de la halle (Fig. 9 et 10). Le niveau d'arasement étant très proche de la surface, un premier nettoyage a rapidement permis de définir un ensemble de

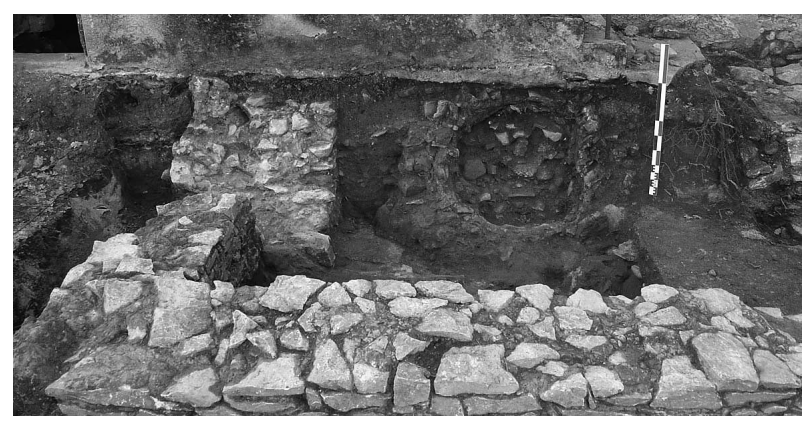

Fig. 9 : Vestige fouillé du four de verrier. Cliché, G. Bonnamour (Arkemine).

murs construits en moellons liés au mortier de chaux. Ils constituent une cellule rectangulaire, intégrant les éléments du four et s'appuyant contre le mur oriental du bâtiment actuel. Malheureusement, seule la partie inférieure d'une moitié du four était conservée, et une tranchée de canalisation orientée nord-sud est venue perturber la zone à une époque plus récente. Toutefois, la chambre du foyer a pu être en partie fouillée. Sa forme est plutôt allongée, proche de celle d'un couloir avec un profil en cuve, et les parois sont recouvertes d'argile rubéfiée. Elle est installée sur un sol stabilisé et ses parois sont constituées de briques, de blocs de calcaire et de grès, le tout lié par une argile sableuse. La sole du four

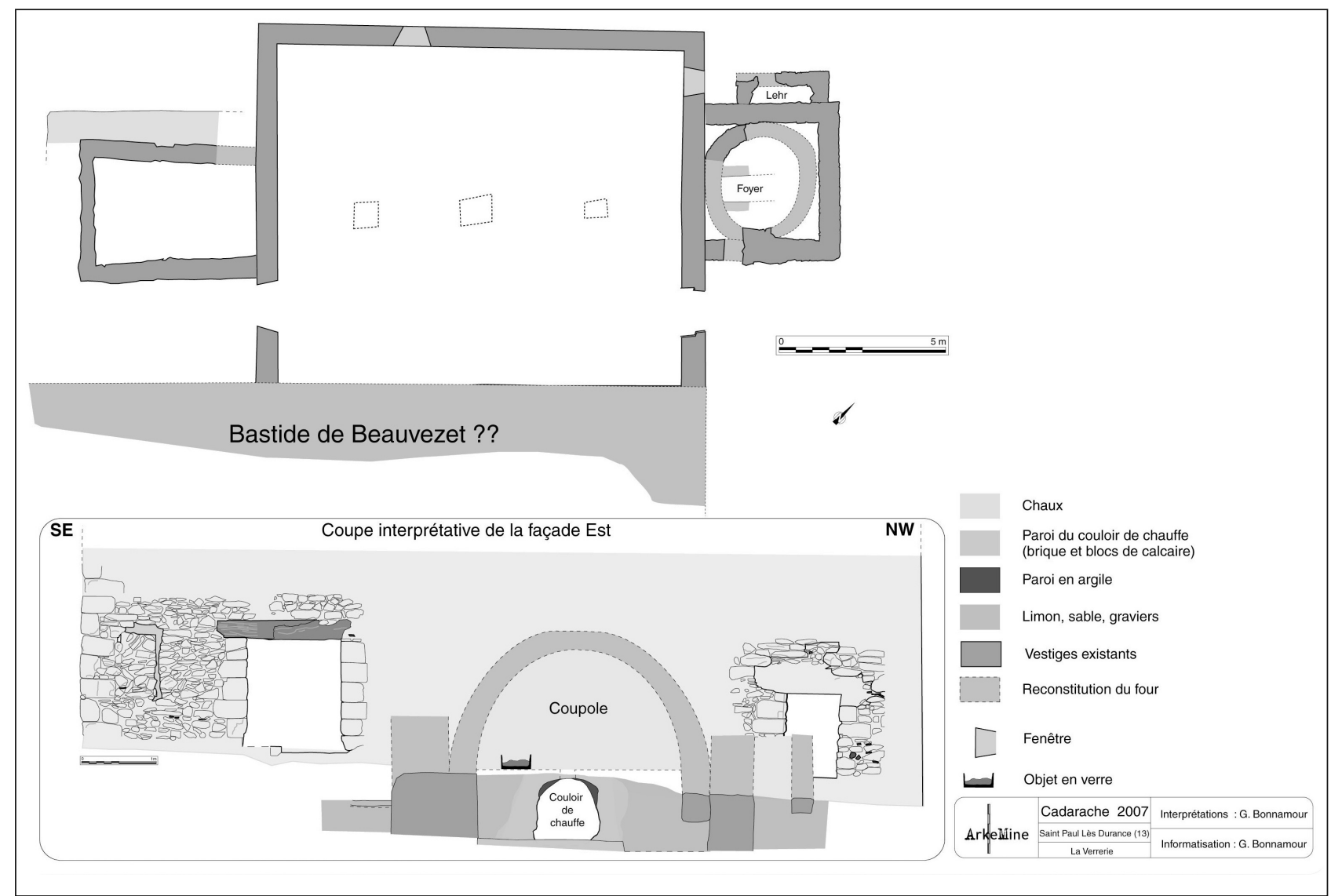

Fig. 10 : Reconstitution de l'atelier de verrier et du four construit contre la façade est. 
a totalement disparu mais quelques fragments ont été découverts. Ils sont constitués d'une argile rouge très cuite recouverte d'une épaisse couche de verre. Une partie de la base du laboratoire de cuisson a également pu être dégagée. Enfin, les fondations d'une petite structure rectangulaire s'appuyant, à l'extérieur et au nord, contre les murs de cette cellule intégrant le foyer, ont été mises au jour.

L'interprétation de la fonction du four, et plus généralement de l'organisation de l'atelier, souffre d'un manque d'information. La partie centrale de ce qui constitue la halle n'ayant pas fait l'objet d'une prescription, elle n'a en effet pas été explorée. Les dimensions et la position du four dans le bâtiment permettent toutefois un rapprochement avec ce qui est connu dans d'autres sites de la même époque, comme à Roquefeuille par exemple (Foy, Vallauri 1989). Dans cet atelier, l'espace central est occupé par un grand four de fusion, organisation qui se retrouve ailleurs dans le Midi de la France (Foy et al. 1983, Commandré, Martin 2009a et b, 2010, Commandré et al. 2011), tandis que plusieurs fours de recuit sont implantés en périphérie. Cette disposition des structures de cuisson existe également dans d'autres ateliers du Midi, comme à Catalo (Commandré, Martin 2008). Elle est également plusieurs fois représentée sur les planches de l'Encyclopédie au XVIII' siècle (Diderot, Le Rond d'Alembert 1772, Petite verrerie à pivette pl. II, Verrerie en bois pl. III et Verrerie en bouteilles pl. VIII). La position du four dans l'atelier de Cadarache rend possible un rapprochement avec les fours de recuit. Il faudrait donc envisager la présence d'un four de fusion au centre de la halle, dans la partie non fouillée. Outre les comparaisons avec d'autres sites, cette hypothèse s'appuie également sur le nombre de verriers en fonction dans l'atelier. Après l'établissement du contrat d'octobre 1667 (34), on compte pas moins de quatre verriers (Antoine et Mechion Desferres, leur oncle Joseph et leur cousin François), assistés d'un tisonnier. La petite taille du four découvert n'étant pas adaptée à ce nombre d'artisans, l'existence d'un plus grand four destiné à la fusion doit être envisagée.

La littérature savante fournit des explications techniques qui plaident en faveur de l'interprétation comme four de recuit. $\mathrm{Au} \mathrm{XVI}^{\mathrm{e}}$ siècle, V. Biringuccio et G. Agricola représentent toujours les fours de fusion au centre de la halle des verriers (Agricola 1556, Biringuccio 1572, fo 65). Ces figures sont d'ailleurs reprises (Fig. 11) au XVIII ${ }^{e}$ siècle par Fl.-A. Néri et C. Merret (Néri et al. 1752, fig. 2). Ce dernier décrit la structure du four de recuit ainsi : « elle a la même forme que le (...) fourneau où l'on fait fondre la matière et où on la tient en fusion; elle comprend deux parties que les Anglois nomment The tower et la Leer. La Tower ou la tour est la partie qui est précisément au-dessus du fourneau de fonte; elle en est séparée par un mur transversal d'un pié d'épaisseur. Au milieu de ce mur de séparation,

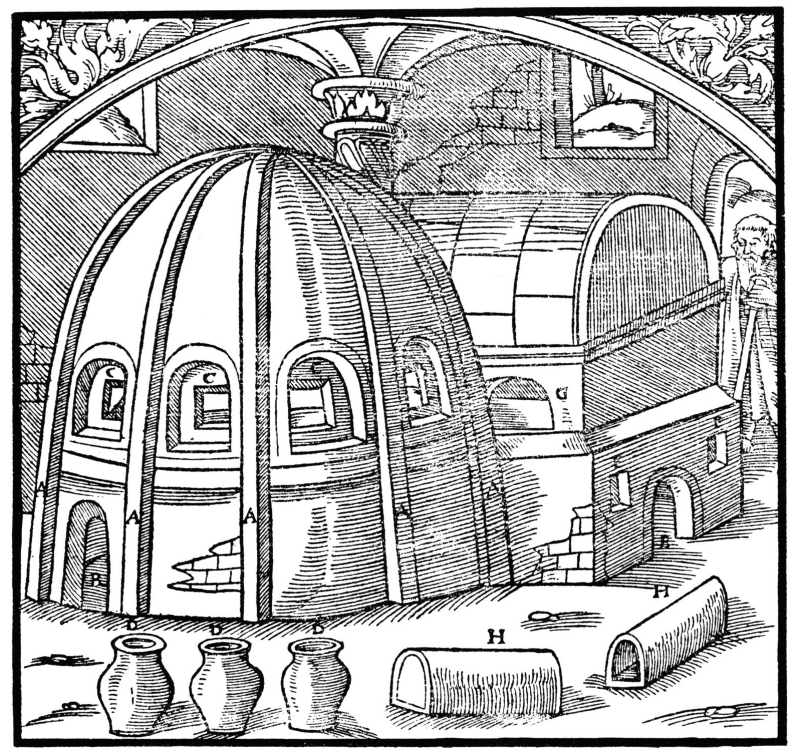

Fig. 11 : Un atelier verrier au $\mathrm{XVI}^{\mathrm{e}}$ siècle d'après G. Agricola : four de fusion en coupole en premier plan, et four de recuit rectangulaire au second plan.

et sur la même ligné que l'ouverture inférieure, se trouve une ouverture ronde par où la flamme et la chaleur entrent dans le fourneau. Imperatus et Agricola font cette ouverture étroite et quarrée : on l'appelle Occhio, l'œil, ou Lumella ; elle est garnie d'un anneau de fer que l'on nomme cavalet ou la couronne ; c'est sur l'aire de cette tour que les verriers mettent leurs vases, lorsqu'ils sont déjà formés pour les faire recuire. Au côté opposé se trouvent deux bouches ou orifices par où les ouvriers mettent les verres avec une fourche sur l'aire de la tour, aussitôt qu'ils sont préparés; et peu après sur des plateaux qui, selon Agricola, doivent être d'argille, qui sont de fer parmi nous et que l'on appelle fraches. Un ouvrier que l'on nomme Saroleman les arrange les uns après les autres par tout la lecra qui a cinq ou six coudées de long, afin qu'ils refroidissent petit à petit, ce qui se fait au bout de la lecra. L'orifice de la lecra vient aboutir dans un endroit où l'on met les verres après qu'ils sont refroidis ; cet endroit se nomme Sarosel, $d u$ nom de celui qui rapporte les fraches de fer dans la lecra » (Néri et al. 1752, XLII).

Les fours décrits dans la littérature et mis au jour par l'archéologie sont généralement hémisphériques, avec un foyer enterré formant un couloir, au-dessus duquel est installée une sole percée d'un trou laissant passé les flammes et la chaleur vers le laboratoire de cuisson. Ce dernier est lui-même recouvert par une coupole, étagée ou non. En suivant les préceptes du De re metallica et de l'Art de la verrerie, une restitution du four de recuit peut donc être proposée (Fig. 10). En admettant la présence d'un four de fusion au centre de l'atelier dans la partie non fouillée, il faut donc imaginer les verriers travaillant d'abord dans ce premier four, avant de transférer les verres, après un premier façonnage, dans le four de recuit

${ }^{34}$ AD Bouches-du-Rhône, 414 E 89, f 703 v (30 octobre 1667). 
situé à portée d'outil, dans un appendice maçonné mais dont le matériau de couverture n'est pas connu. Ce four est alimenté en combustible par une entrée basse (Fig. 10), qui, certainement, s'ouvrait du côté de l'atelier au ras du sol, en prolongement du couloir où était installé le foyer. Quant à l'espace adjacent au four au nord, il peut tout à fait correspondre à la lecra (ou leer, ou encore lehr), où se termine donc le refroidissement des verres mis en forme.

Quelques zones d'ombre subsistent autour de ce four. Tout d'abord, aucune ouverture n'a été mise en évidence dans le mur entre la halle et l'espace restitué de la chambre supérieure du four. Ensuite, alors que la majorité des fragments de creusets ont été découverts à l'ouest de la fouille, à l'opposée du four, quelques-uns proviennent tout de même du secteur du four. Ceux-ci, servant à faire fondre le verre, sont normalement utilisés dans les fours de fusion. Toutefois, dans de nombreux ateliers, les creusets ont été découverts en dehors des fours, témoignant de perturbations dans la répartition spatiale des artefacts liés à la production au moment de l'abandon par les verriers (Commandré, Martin 2008). Les creusets pouvaient être écaillés pour récupérer le verre piégé sur les parois et recyclés pour la fabrication d'autres creusets. À Cadarache, ces opérations étaient peut-être réalisées dans la partie occidentale de la halle.

\section{Production et organisation du travail}

\section{Les matières premières}

Le sous-arrentement de 1670 renseigne sur l'outillage et les matières premières. En effet, pour faire fonctionner l'atelier, François Descrivas reçoit du matériel constitué de soude, verre cassé, canes, marteaux, barres de feu et autres (35), ainsi qu'un fourneau et six cruzeaux remplis de verre estant dans icelluy, estimés à quattre cens livres (36). Il reçoit également la somme de 1964 livres et 3 sous, et achète à Melchion Desferres un mulet, un sallet (housse d'une mule de charrette) et un crible pour cribler le verre (37). La fouille du site est venue compléter ces informations. Ainsi, les matières premières utilisées sont :

- La silice : c'est l'élément fondamental pour la fabrication du verre. Le lit de la Durance, qui se trouve à proximité, est riche en alluvions intégrant des sables, des graviers et des galets de quartz. Le sable est privilégié par les verriers, car plus facilement utilisable.

- La soude : elle n'est pas produite sur place, mais sa fabrication est très répandue en Provence et Languedoc à partir de plantes halophiles comme la salicorne, et plus particulièrement en Camargue et autour de l'Étang de Berre (Amouric, Foy 1985 et 1992 ; Foy 1988, 36 ; Amouric 1993). Elle est transportée sous la forme de " pierres à soude » ou de cendres et sert de fondant pour les verriers dans le but d'abaisser le point de fusion de la silice.

- La chaux : plusieurs fours à chaux sont connus au travers des registres des notaires dans le courant du $\mathrm{XVII}^{\mathrm{e}}$ siècle à Cadarache (38), et le diagnostic préventif en avait mis un autre au jour, datant du XVIII' ${ }^{\mathrm{e}}$ siècle. Par ailleurs, des amas de chaux ont été dégagés au moment de la fouille à l'ouest du bâtiment. Il s'agit là encore d'un élément fondamental dans le processus de fabrication du verre. Car, bien que nécessaire, l'utilisation d'un fondant tel que la soude peut fragiliser certaines liaisons chimiques du verre. Intégrée dans la composition du verre, la chaux permet alors de palier ce problème car elle augmente sa résistance, son éclat, et diminue sa solubilité. Pourtant, pendant longtemps, les verriers n'avaient visiblement pas conscience de ce rôle important. La chaux étaient introduite dans le processus de fabrication par l'intermédiaire d'autres éléments, comme la sable ou les cendres alcalines (Foy 1988, 40). Au $\mathrm{XVII}^{\mathrm{e}}$ siècle, il n'est pas certain que les artisans aient assimilé cette partie du savoir-faire.

- Pour le groisil : il est généralement acheté ou échangé à des marchands. Par recyclage, les déchets issus de la fabrication et de la mise en forme des verres à Beauvezet ont alimenté les réserves de cette matière première, comme en témoigne la mention de verre cassé dans les archives.

\section{Outillage et répartition des tâches}

De nombreux fragments de creusets ont été découverts. Sur l'ensemble du lot, une grande variabilité des épaisseurs de lèvres a été constatée. Il a été possible, à partir de quelques échantillons, de proposer une reconstitution de la forme et des dimensions de l'un des creusets utilisés à la verrerie de Cadarache (Fig. 12). Il s'agit d'un récipient d'une quarantaine de centimètres de diamètre et d'une trentaine de centimètres de profondeur. L'épaisseur de la pâte varie de deux centimètres au niveau du bord à cinq centimètres pour le fond $\mathrm{du}$ creuset. La pâte est de couleur grise parfois légèrement brique, à fin dégraissant et la mise en forme doit se faire par boudinage. Les parois sont vitrifiées à l'intérieur, mais également à l'extérieur du creuset. Sur quelques échantillons caractéristiques de fond de creuset, des fragments de verre sont visibles. Les creusets sont généralement posés directement sur la sole du four pour permettre aux artisans de fondre la matière vitrifiable jusqu'à obtenir la pâte malléable que les verriers pourront façonner. Si l'archéologie n'a pas pu déterminer le nombre de creusets en usage à Cadarache, les archives ont apporté plus d'informations. En 1671, des contrats d'arrentements témoignent de la présence de six creusets dans l'atelier des verriers.

\footnotetext{
${ }^{35}$ AD Bouches-du-Rhône, 399 E 319, fo 483 v $^{\circ}$ (20 septembre 1670).

${ }^{36}$ AD Bouches-du-Rhône, 399 E 319, fo $606 \mathrm{v}^{\circ}$ (17 avril 1671).

${ }^{37}$ AD Bouches-du-Rhône, 399 E 319 , fo $512 \mathrm{v}^{\circ}$ (14 octobre 1670).

${ }^{38}$ AD Bouches-du-Rhône, 399 E 319, fo 583 (8 mars 1671).
} 


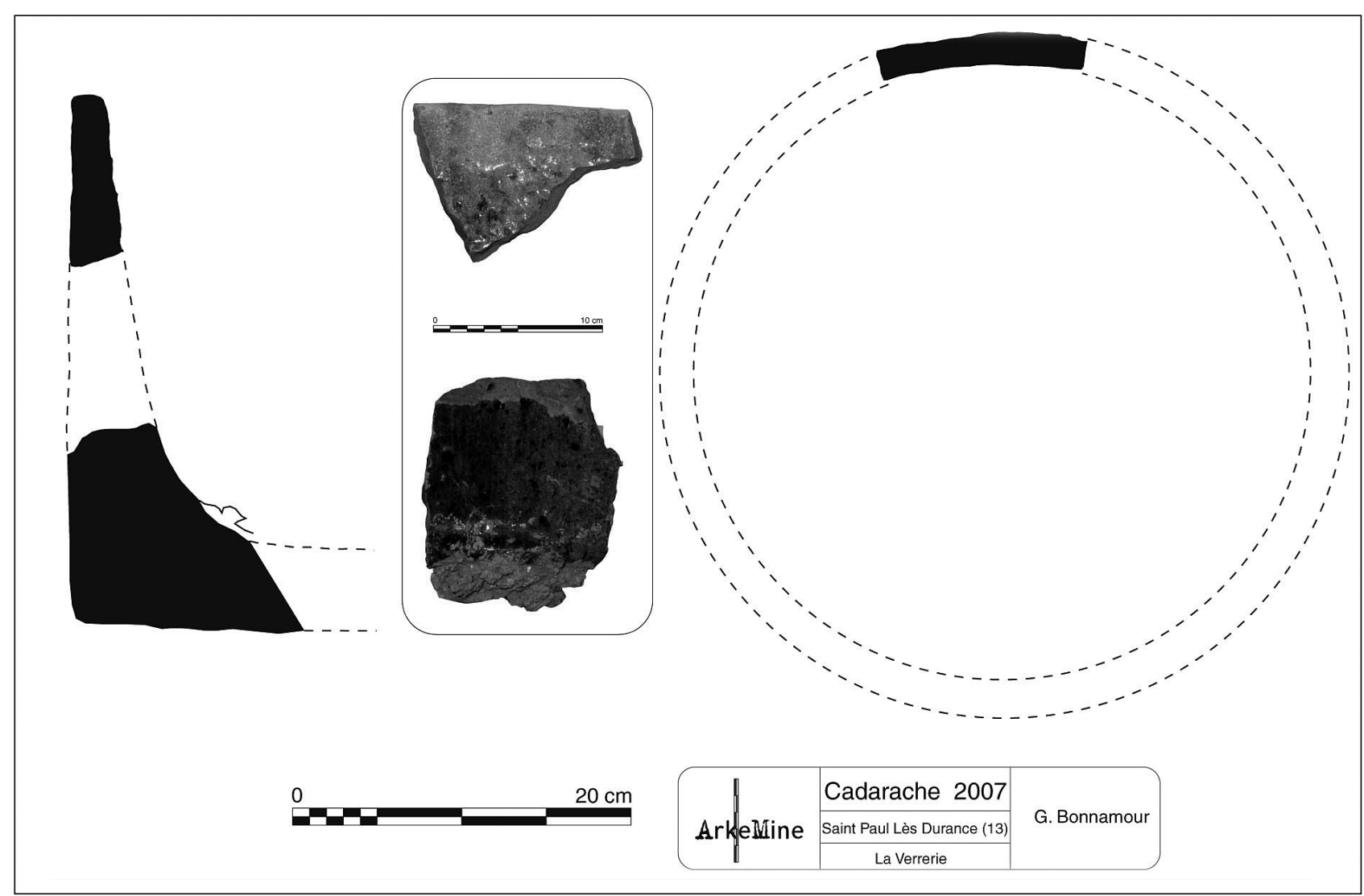

Fig. 12 : Proposition de reconstitution du creuset.

Plusieurs autres outils sont utilisés. Ils sont mentionnés par les sources écrites, ou, parfois, c'est l'étude du mobilier qui atteste de leur utilisation :

- Les cannes de souffleur permettent aux verriers de récupérer au sein du creuset la pâte de verre malléable et de façonner les objets. Plusieurs cannes sont nécessaires pour un seul verrier. Des pontils devaient également être présents, mais ne sont pas mentionnés. Ils servent à manipuler les pièces soufflées et à procéder à l'ajout d'autres paraisons.

- Les marteaux permettent de casser le groisil et les pierres de soude. Enfin, la présence d'un crible pour préparer le groisil témoigne du soin porté à la préparation du verre composant le mélange qui va subir la fusion.

- Les pinces sont utilisées pour le façonnage des verres, et notamment des paraisons. Elles laissent des déchets pincés, dont plusieurs ont été retrouvés lors de la fouille.

- Les barres de feu servaient certainement à attiser et remuer le feu du foyer du four.

Ces outils révèlent également la répartition des tâches au sein de l'atelier. Les maîtres-verriers s'occupaient de la préparation des matières premières et du mélange introduit dans le creuset, mais également de la mise en forme et du façonnage des objets. La gestion du feu incombait aux tisonniers, véritables ouvriers spécialisés, qui sont mentionnés à deux reprises dans les archives. À ces deux fonctions s'ajoutaient un « homme de travail » chargé de couper le bois dans la forêt et de le livrer à la verrerie.

\section{Production et commercialisation}

La fouille a mis au jour de nombreux déchets de production, répartis en plusieurs catégories : fragments en forme de corne, coulées, fils de verre, ampoules et déchets portant des traces de pincement (Fig. 13). Certains d'entre eux témoignent des étapes de la chaîne opératoire technique. La fabrication des anses produit les déchets en forme de fil de verre, tandis que les traces de pincement trahissent l'utilisation de pinces métalliques. Les couleurs varient entre le vert, l'azur et le jaune terne.

Peu de verres façonnés ont été découverts, et aucune forme n'a pu être déterminée. Les fragments mis au jour peuvent provenir de ratés de cuisson, d'objets finis mais cassés ou de verres récupérés.

La question de la production et de la commercialisation est également difficile à aborder par les sources historiques. Rien n'est dit sur les formes produites, les actes évoquant au mieux des verres (39) et leur emballage sans plus de précision. Les Desferres entretiennent des relations avec des marchands verriers d'Aix (40) en

\footnotetext{
${ }^{39}$ AD Bouches-du-Rhône, 399 E 319, $\mathrm{f}^{\circ} 440 \mathrm{v}^{\circ}$ (2 juin 1670) ; 320, fo $38 \mathrm{v}^{\circ}$ (17 juin 1674).

${ }^{40}$ AD Bouches-du-Rhône, 399 E 319, fo 399 (26 février 1670).
} 


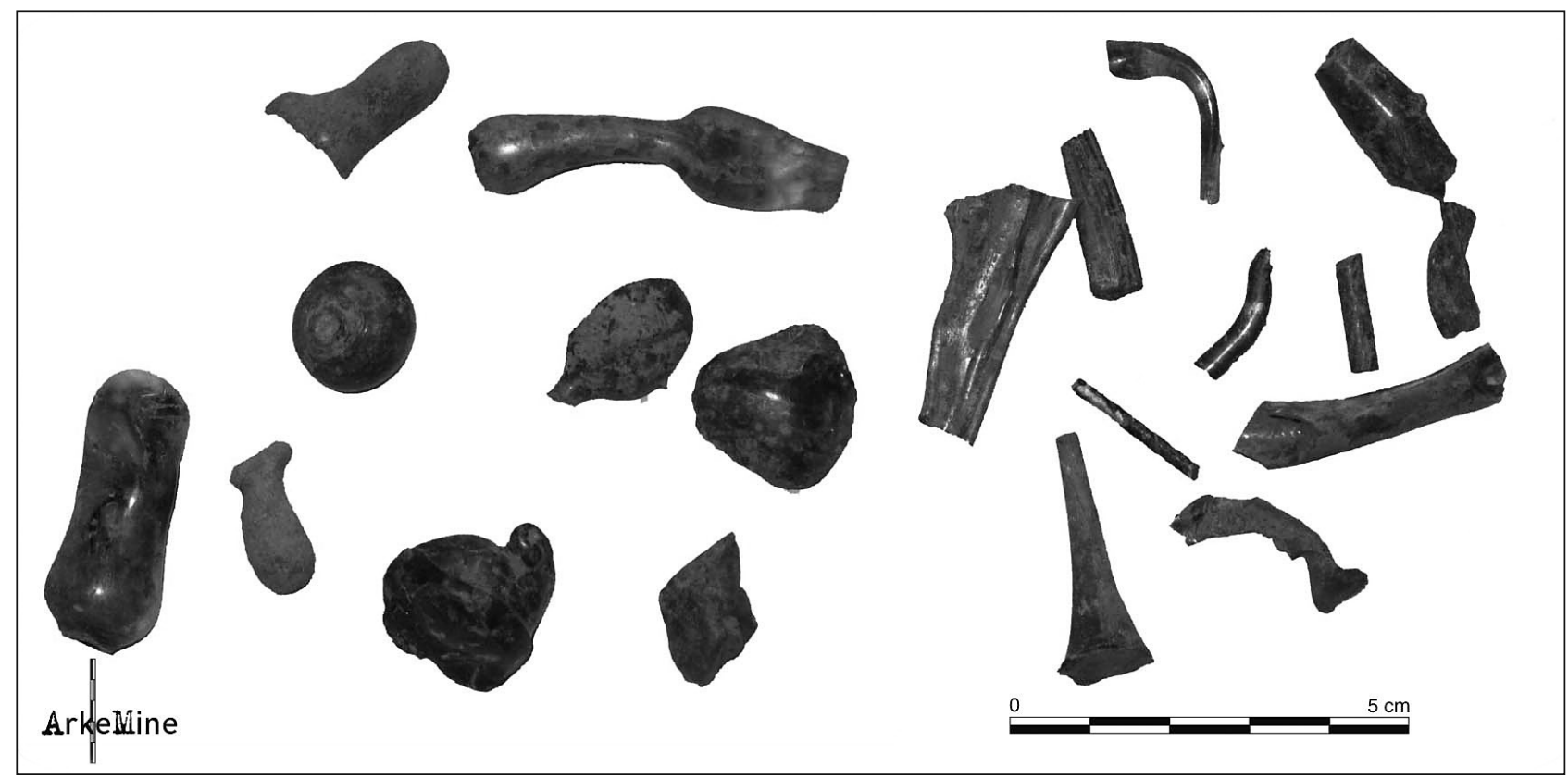

Fig. 13 : Fragments de déchets de fabrication. Cliché, G. Bonnamour (Arkemine).

basse Provence, ainsi que de Ribiers (41) et SaintChristol (42) en moyenne vallée de la Durance, ce qui marque une diffusion régionale de la production.

\section{En guise de conclusion}

La confrontation des informations issues de la fouille archéologique, des relevés du bâti et du dépouillement des sources historiques permet de dresser un portrait relativement précis de la bastide de Beauvezet de Cadarache entre la fin du Moyen Age et la fin du $\mathrm{XVII}^{\mathrm{e}}$ siècle. Peut-être issue d'une volonté seigneuriale de mettre en valeur un terroir déserté depuis le $\mathrm{XIV}^{\text {e }}$ siècle, la bastide de Beauvezet est bâtie sur le modèle de nombre d'établissements similaires connus en Provence par les textes. Ce modèle est à la fois architectural, comme en témoigne la fenêtre à meneau conservée, et fonctionnel. Installée à la limite entre terroir cultivé et incultum, la bastide est ici un établissement pionnier tourné principalement vers la céréaliculture et le pastoralisme. Pour la première fois, l'archéologie met en évidence la perpétuation de ce système médiéval de mise en valeur pendant une bonne partie de l'époque moderne.

La bastide de Beauvezet a subi des modifications au moment de l'installation des verriers à Cadarache, attirés par la proximité de certaines ressources. Le bâtiment en pierres, en élévation au moment de la fouille, s'appuie contre un mur d'origine de la bastide de Beauvezet. La fouille a mis au jour les vestiges d'un four de recuit, et l'organisation spatiale rappelle celle de plusieurs ateliers contemporains de celui de Cadarache et découverts dans le Midi de la France.

L'atelier, dont la diffusion des productions semble régionale, disparaît probablement après 1676 puisqu'aucun acte le concernant n'a par la suite été retrouvé. Pourtant, l'activité verrière a modifié durablement la configuration de la bastide de Beauvezet. Malgré une dizaine d'années d'activité verrière à la fin du $\mathrm{XVII}^{\mathrm{e}}$ siècle, le toponyme la Verrerie supplante celui de la bastide.

\section{Remerciements}

Nous remercions vivement D. Foy (DR CNRS, CCJUMR 7299), I. Commandré (Doctorante, CCJ-UMR 7299) et J. Thiriot (DR honoraire, LA3M-UMR 7298) pour les relectures et pour leurs conseils très avisés.

\section{Sources imprimées et savantes}

Agricola 1556 : AGRICOLA (G.) et HOOVER (H. Cl.) et HOOVER (L. H.) (trad.), De re mettalica, NewYork, Dover Publications Inc., 1950, 638 p.

Albanès 1895 : ALBANES (J.-H.), Gallia christiana novissima. Histoires des archevêchés, évêchés et abbayes de France. Province d'Aix, Montbéliard, Imprimerie valentinoise, 1895, $616 \mathrm{p}$.

Biringuccio 1572 : BIRINGUCCIO (V.), La pyrotechnie, ou art du feu contenant dix livres ausquels est amplement traicté et diversité des minières, fusions et séparations des métaux, des formes et moules pour jetter artilleries, cloches et toutes autres

\footnotetext{
${ }^{41}$ AD Bouches-du-Rhône, 399 E 320, $\mathrm{f}^{\circ} 38 \mathrm{v}^{\circ}$ (17 juin 1674).

${ }^{42}$ AD Bouches-du-Rhône, 399 E 319, fo $440 \mathrm{v}^{\circ}$ (2 juin 1670).
} 
figures ; distillations, des mines... chez C. Frémy, Paris, 1572, $168 \mathrm{f}$.

Guérard 1857 : GUERARD (B.), Cartulaire de l'abbaye de Saint-Victor de Marseille. Collection des cartulaires de France, t. VIII, Paris, typographie de Ch. Lahure, 1857, 2 vol., CLVI-651, 944 p.

Diderot, Le Rond d'Alembert 1772 : DIDEROT (D.), LE ROND D'ALEMBERT (J.), Recueil de planches sur les sciences, les arts libéraux et les arts méchaniques, avec leur explication, vol. IX, Paris, chez Briasson, 1772, $337 \mathrm{pl}$.

Néri et al. 1752 : NÉRI (Fl. A.), MERRET (Chr.), ORSCHALL (J. Chr.), L'art de la verrerie, Paris, chez Durand et Pissot, 1752, 629 p.

\section{BIBLIOGRAPHIE}

Amouric 1993 : AMOURIC (H.), Calissane et Merveille ; deux domaines dans l'économie de l'étang de Berre (de la fin du Moyen Age au XVIII ${ }^{\mathrm{e}}$ siècle). Archéologie et environnement : de la Sainte-Victoire aux Alpilles (LEVEAU (Ph.), PROVANSAL (M.) dir.), Aix-en-Provence, Presses de l'Université de Provence, 1993, p. 315-373.

Amouric, Abel 1995 : ABEL (V.), AMOURIC (H.), Les ateliers de l'Huveaune à l'époque moderne. Actes $d u$ $5^{e}$ colloque sur la céramique médiévale. Rabat, 11-17 novembre 1991, Rabat, Institut national des sciences de l'archéologie, 1995, 84-94.

Amouric, Foy 1985 : AMOURIC (H.), FOY (D.), Notes sur la production et la commercialisation de la soude dans le Midi méditerranéen du XIII ${ }^{\mathrm{e}}$ au XVIII ${ }^{\mathrm{e}}$ siècle. Histoire des techniques et sources documentaires, méthodes d'approche et expérimentation en région méditerranéenne. Cahier $n^{\circ} 7$, actes du colloque $d u$ G.I.S., Aix-en-Provence, 21-23 octobre 1982, Aix-enProvence, 1985, p. 157-171.

Amouric, Foy 1992 : AMOURIC (H.), FOY (D.), Les artisanats de la céramique et du verre en Provence : la question du combustible au Moyen Age et à l'époque moderne. Protoindustries et histoire des forêts. Les Cahiers de l'ISARD 3, 1992, p. 45-61.

Amouric et al. 1999 : AMOURIC (H.), RICHEZ (F.), VALLAURI (L.), Vingt mille pots sous les mers. Le commerce de la céramique en Provence et Languedoc, $X^{e}-X I X^{e}$ s., Catalogue de l'exposition, Aix-en-Provence, Edisud, 1999, 197 p.

Bernardi 1996 : BERNARDI (Ph.), De la forme nouvelle aux nouvelles applications de la forme : une brève histoire de la croisée en Provence. in : L'innovation technique au Moyen Age. Actes du VI Congrès international d'archéologie médiévale. $1^{e r}$. 5 octobre 1996, Dijon-Mont Beuvray-Chenôve-Le Creusot-Montbard. Société d'Archéologie Médiévale, 1996, 224-229.

Bonnamour et al. 2009 : BONNAMOUR (G.), TISSOT (M.), DORMOY (C.), VASCHALDE (C.),
Cadarache 2007, La Verrerie (Saint-Paul-LèsDurance, 13), Vol. 1/2, Rapport de fouille d'archéologie préventive, Arkemine, 2009, 245 p.

Bonnamour 2007 : BONNAMOUR (G.), La Verrerie, Cadarache (Saint-Paul-Lès-Durance, 13), Bilan scientifique régional PACA, SRA PACA, 2007, p. 166.

Commandré, Martin 2008 : COMMANDRE (I.), MARTIN (Fr.), Les verreries modernes dans la région de la Montagne Noire : présentation des premiers résultats avec les ateliers de Catalo (Hérault) et de Cantesoubre (Tarn), Bulletin de l'Association française pour l'archéologie du verre 2008, p. 88-98.

Commandré, Martin 2009a : COMMANDRÉ (I.), MARTIN (Fr.), Fraïsse-sur-Agoût (Héraut). Verrerie du Bureau, Archéologie médiévale 39, 2009, p. 332.

Commandré, Martin 2009b : COMMANDRÉ (I.), MARTIN (Fr.), Lacabarède (Tarn). Verrerie de Candesoubre, Archéologie médiévale 39, 2009, p. 332 .

Commandré 2010 : COMMANDRÉ (I.), MARTIN (Fr.), Lacabarède (Tarn). Candesoubre, Archéologie médiévale 39, 2009, p. 317-318.

Commandré et al. 2011 : COMMANDRÉ (I.), MARTIN (Fr.) dir., RIOLS (A.), GRATUZE (B.), POUYET (E.), ROULEAU (R.) coll., Une verrerie moderne dans les Monts du Somail (Hérault) : l'atelier forestier du Bureau au XVII ${ }^{e}$ s. (commune de Fraïsse-sur-Agoût), Etudes héraultaises 41, 2011, p. $1-15$.

Commandré en cours : COMMANDRÉ (I.), Archéologie et histoire de l'artisanat verrier en Languedoc (FOY (D.) dir.), Université d'AixMarseille-Centre Camille Jullian UMR 7299, thèse en cours.

Coulet 1988 : COULET (N.), Aix-en-Provence. Espace et relations d'une capitale (milieu XIV ${ }^{e}$ s.-milieu XV s.), Aix-en-Provence, Publications de l'université de Provence, 1988, 2 vol., 1238 p.

Drendel 1991 : DRENDEL (J. V.), Economy and society in a medieval provençal town : Trets 1296-1347, Thèse de doctorat dactylographiée soutenue à l'Université de Toronto (Canada), 1991, 512 p.

Foy 1988 : FOY (D.), Le verre médiéval et son artisanat en France méditerranéenne, Paris, éd. du CNRS, 1988, $467 \mathrm{p}$.

Foy et al. 1983 : FOY (D.), AVEROUS (J.-C.), BOURREL (B.), Peyremoutou : une verrerie du $\mathrm{XVII}^{\mathrm{e}}$ siècle dans la Montagne Noire, Archéologie $d u$ Midi médiéval 1, 1983, p. 93-102.

Foy et al. 1989 : FOY (D.), VALLAURI (L.), Roquefeuille, une verrerie provençale aux $\mathrm{XVII}^{\circ}$ et XVIII ${ }^{\mathrm{e}}$ siècle, Atelier de verriers de l'antiquité à la période préindustrielle, (Rouen, 24-25 nov.1989), AFAV, 1991, p.139-152. 
Martin 2007 : MARTIN (L.), Rapport intermédiaire de diagnostic, chantier Iter, Rapport intermédiaire de diagnostic archéologique préventif, INRAP / SRA Provence-Alpes-Côte d'Azur, 2007.

Martin, Fournier 2007 : MARTIN (L.), FOURNIER (St.), Saint-Paul-lès-Durance, I.T.E.R.-Cadarache, Bilan scientifique régional PACA, SRA PACA, 2007, p. 166.

Moulin 1910 : MOULIN (P.), Documents relatifs à la vente des biens nationaux, département des Bouchesdu-Rhône, Marseille, Barlatier, 1910, 4 vol., 592674-647-581 p.

Mouton 2008 : MOUTON (D.), Mottes castrales en Provence : les origines de la fortification privée au Moyen Age. DAF 102, Paris, éd. de la Maison des Sciences de l'Homme, 2008, 148 p.
Vaschalde 2008 : VASCHALDE (C.), Rapport de dépouillement des registres des notaires de SaintPaul-Lès-Durance et Meyrargues (13), in : BONNAMOUR (G.), TISSOT (M.), DORMOY (C.), VASCHALDE (C.), Cadarache 2007, La Verrerie (Saint-Paul-Lès-Durance, 13), Vol. 2/2 Rapport de fouille d'archéologie préventive, Arkemine, 2009.

Vaschalde en cours : VASCHALDE (C.), Chaufournerie, charbonnage, artisanats du feu et ressources naturelles en basse Provence médiévale. Approches croisées archéologiques, historiques et ethnoarchéologiques, thèse en cours (DURAND (A.) et THIRIOT (J.) dir.), Aix-Marseille Université. 